\title{
AGRICULTURA FAMILIAR: PERSPECTIVAS DE DESARROLLO RURAL EN EL MUNICIPIO DE SAN MIGUEL.
}

\section{FAMILY FARMING: PERSPECTIVES OF RURAL DEVELOPMENT IN THE MUNICIPALITY OF SAN MIGUEL}

Felipe Santiago Salvador Bonilla

Ingeniero Agrónomo

Investigador | Universidad de Oriente

fsalvador@univo.edu.sv

\section{Resumen}

El objetivo principal de este trabajo fue analizar las prácticas de agricultura familiar y las condiciones de vida en comunidades La Canoa, San Jacinto, El Volcán y El Brazo, del municipio de San Miguel, El Salvador, considerando la importancia de las actividades agropecuarias para el desarrollo comunitario. Se utilizó un diseño exploratorio sobre un tema desconocido o poco estudiado, por lo que sus resultados constituyen una visión aproximada del fenómeno. El diseño fue explicativo para entender como las prácticas de agricultura familiar mejoran las condiciones de vida en las familias. Los resultados evidenciaron un sistema agropecuario basado en granos básicos, particularmente maíz, que limita la diversidad de la producción. Se definió que existe un índice bajo de asistencia a la producción, de igual, forma un índice bajo en iniciativas de producción agropecuaria. La Escala Latinoamericana y Caribeña de Seguridad Alimentaria (ELCSA) determinó apenas un $8.7 \%$ de familias que se encuentran en seguridad alimentaria, un $34.6 \%$ en inseguridad leve, un 22.8 en inseguridad moderada y un $33.9 \%$ en inseguridad severa. El desarrollo rural en las comunidades está asociado a los perfiles de medios de vida, por tanto, un débil sistema agropecuario, caracterizado por un bajo índice de iniciativas agropecuarias, da paso a condiciones de inseguridad alimentaria, deteriorando la calidad de vida de las familias en el área rural del municipio de San Miguel.

El desarrollo rural en las comunidades está asociado a los perfiles de medios de vida. Por tanto, un débil sistema agropecuario, caracterizado además por un bajo índice de iniciativas agropecuarias, da paso a condiciones de inseguridad alimentaria, deteriorando la calidad de vida de las familias en el área rural del municipio de San Miguel.

Palabra clave: Agricultura familiar, seguridad alimentaria y nutricional, desarrollo rural, condiciones de vida. 


\section{Abstract}

The main purpose of this study was to analyze the family farming practices and the living conditions in the communities of La Canoa, San Jacinto, El Volcán and El Brazo, from the municipality of San Miguel, considering the importance that the farming activities for the community development has. An exploratory approach of this unknown topic was used, so the results are an approximated vision of this phenomenon. Besides, an explanatory design was applied to understand how the family farming practices improve the family living conditions. Results showed a farming system in the rural areas based in basic grains, mainly corn grains, which limits the production diversity. A low index of production support was defined, as well as a low index in farming production initiatives. The Latin American and Caribbean Food Security Scale (ELCSA) roughly determined that $8.7 \%$ of the families are ensured food security, 34.6\% are in light food insecurity, 22.8 are in mild food insecurity, and $33.9 \%$ are in severe food insecurity. Rural development is associated to the livelihood profiles. Therefore, a weak farming system characterized by a low index of farming initiatives lead out to food insecurity conditions, damaging the living conditions of the families from the rural area of the municipality of San Miguel.

Keywords: Family farming, food and nutritional insecurity, rural development, living conditions.

\section{Introducción}

Los problemas que afectan a la humanidad son diversos y complejos, sin omitir que existen avances y desarrollo de muchos campos de las ciencias que han contribuido a generar mucha riqueza en el planeta. Sin embargo, ello no ha llevado a erradicar el hambre, como lo expone la Organización de las Naciones Unidas para la Alimentación y la Agricultura (FAO), el Fondo Internacional de Desarrollo Agrícola (FIDA), la Organización Mundial de la Salud (OMS), el Programa Mundial de Alimentos (PMA) y el Fondo de las Naciones Unidas para la Infancia (UNICEF), en su informe: El estado de la Seguridad Alimentaria y la Nutrición en el mundo 2018 , señala que en el año 2018 "Los nuevos datos siguen indicando un aumento del hambre mundial en los últimos años, después de un período de disminución prolongado. Se estima que 821 millones de personas aproximadamente, una de cada nueve personas en todo el mundo una de cada nueve personas en todo el mundo están subalimentadas, (p. 2).

Según Esquinas (2017), las causas fundamentales de la Inseguridad Alimentaria (en adelante 
InSAN) en el mundo, se deben a la especulación de precios en los alimentos. Para combatir tales causas propone la producción de alimentos a nivel local, a la cual se ha denominado Agricultura Familiar, como estrategia necesaria para avanzar en Seguridad Alimentaria y Nutricional en los países donde los indicadores de nutrición no alcanzan parámetros adecuados.

En sí el papel de la agricultura familiar está indisociablemente vinculada a la SAN. En los países en desarrollo, como en los desarrollados, la agricultura familiar es la forma agrícola predominante en el sector de la producción alimentaria, y sus prácticas están a la base de producción a pequeña escala, como parcelas de hortalizas, granos básicos y pequeñas especies. Además, y como valor agregado, cabe mencionar que la agricultura familiar está asociada a buenas prácticas agrícolas, estrategias de organización comunitaria, lucha contra el hambre y promoción de la igualdad de género. Los últimos dos factores están vinculados a dos objetivos de desarrollo sostenible (FAO, 20I4; ONU, 20I7).

Partiendo del anterior enfoque, el gobierno de El Salvador desde el año 201 I implementó el Plan de Agricultura Familiar (PAF), para buscar erradicar el hambre en la población y mejorar la SAN. Bajo esa dinámica, diversas iniciativas agropecuarias se han desarrollado en las comunidades rurales. Por ejemplo, pequeñas unidades productivas de granos básicos, hortalizas y especies menores con el apoyo del Ministerio de Agricultura y Ganadería (MAG) y otras instituciones no gubernamentales, que han colaborado para introducir la agricultura familiar.

En resumen, el estudio buscó analizar si las comunidades que han puesto en práctica las técnicas de agricultura familiar están mejorando los niveles de seguridad alimentaria y condiciones de vida, con la finalidad de hacer un análisis riguroso de aquellos aspectos de connotación política, económica y social que llevan al hombre y la mujer de comunidad a luchar por el derecho a una alimentación adecuada. En ese sentido la investigación tendrá un enfoque cuantitativo.

\section{Metodología}

El estudio fue de corte transversal con un diseño exploratorio y explicativo debido a que se estudió si las comunidades que realizan prácticas de agricultura familiar mejoran las 
condiciones de vida (Hernández Sampieri y otros, 2014). Además, de medir variables, se estudiaron las relaciones de influencia entre ellas para conocer la estructura y los factores que intervienen en los fenómenos sociales y su dinámica (Sierra Bravo, 200I). Las comunidades que participaron en el estudio fueron: La Canoa, San Jacinto, El Volcán y El Brazo, todas del municipio de San Miguel, con una población de 400 familias; se trabajó con una muestra de 197 familias, calculada a través de la fórmula para poblaciones finitas a un nivel del $95 \%$ de confianza y un error muestral del $5 \%$.

Se plantearon las siguientes hipótesis:

Hi: Los niveles de Seguridad Alimentaria mejoran a partir de Prácticas de Agricultura Familiar en hogares de cuatro comunidades: La Canoa, San Jacinto, El Volcán y El Brazo.

Hi: Las Iniciativas de Prácticas de Agricultura Familiar y otros factores externos determinan las Condiciones de Vida en hogares de cuatro comunidades: La Canoa, San Jacinto, El Volcán y El Brazo.

El método de recolección de información fue a través de la encuesta, mediante la aplicación de un cuestionario dirigido a jefes y jefas de hogar, que forman parte de algún programa vinculado a la agricultura familiar desarrollado por instituciones gubernamentales u ONG's, en las comunidades seleccionadas. Para el tratamiento estadístico de la información obtenida a través de la aplicación del cuestionario se utilizaron los siguientes programas informáticos:

a) PSPP (2016): Mediante este software se elaboró la base de datos con la información obtenida a través del cuestionario. Posteriormente se procedió a realizar varias tablas de análisis univariado y bivariado. La prueba de hipótesis se realizó mediante el estadístico Chicuadrado para determinar significancia en relaciones lógicas de carácter nominal. De igual manera, se construyeron índices sumatorios ponderados, utilizando la metodología de Padua y otros (1979) asignando pesos diferenciados a las alternativas de respuestas en los indicadores que se desea tengan valores mayores. Ello permitió realizar una ponderación no sobre los valores originales, sino sobre algunas alternativas de respuesta en particular que facilitó las pruebas de hipótesis.

b) Microsoft Word (versión 2016): Se utilizó para la redacción de la investigación, y mejoramiento de las tablas de resultados.

c) Microsoft Excel (versión 2016): Se utilizó para la construcción de tablas y gráficos 
obtenidos mediante el software estadístico PSPP.

\section{Resultados}

\section{"Características sociodemográficas en comunidades}

En este apartado, se presentan las características sociodemográficas de 197 familias que fueron parte del estudio. Están distribuidas en cuatro comunidades del municipio de San Miguel. Estas familias trabajan en iniciativas agropecuarias con un enfoque de agricultura familiar.

Tabla I. Características sociodemográficas en comunidades.

\begin{tabular}{|c|c|}
\hline Descripción de atributos & Porcentaje \\
\hline \multicolumn{2}{|c|}{ Género } \\
\hline Masculino & $61.4 \%$ \\
\hline Femenino & $38.6 \%$ \\
\hline \multicolumn{2}{|c|}{ Procedencia del encuestado } \\
\hline La Canoa & $21.3 \%$ \\
\hline San Jacinto & $24.9 \%$ \\
\hline El Volcán & $21.8 \%$ \\
\hline El Brazo & $32.0 \%$ \\
\hline \multicolumn{2}{|c|}{ Número de miembros en hogares } \\
\hline I a 2 miembros & $13.2 \%$ \\
\hline 3 a 4 miembros & $43.1 \%$ \\
\hline 5 a 6 miembros & $33.0 \%$ \\
\hline Más de 6 miembros & $10.7 \%$ \\
\hline \multicolumn{2}{|c|}{ Nivel de escolaridad } \\
\hline Ninguno & $33.5 \%$ \\
\hline Educación básica & $59.9 \%$ \\
\hline Educación media & $5.6 \%$ \\
\hline Educación superior & $1.0 \%$ \\
\hline
\end{tabular}

Fuente: Elaboración propia

En cuanto a la distribución de la variable género, fueron entrevistados $61.4 \%$ jefes de hogares y $38.6 \%$ jefas de hogares. Respecto a la muestra por comunidad, el menor porcentaje, $21.8 \%$, corresponde a El Volcán y el mayor porcentaje, $32.0 \%$, corresponde a la comunidad El Brazo. Se preguntó por el tamaño del grupo familiar, resultando mayor el rango de 3 a 4 miembros con un $43.1 \%$, y en menor porcentaje con $10.7 \%$ más de 6 miembros. En cuanto al nivel de escolaridad, se encontró el $59.9 \%$ con educación básica, un $33.5 \%$ con ningún grado escolar, un $5.6 \%$ con educación media y un $1.0 \%$ con educación superior.

\section{"Sistema de producción agropecuaria}

Este apartado muestra los rubros de producción agropecuaria en las comunidades, 
exceptuando los cultivos de exportación como el café y la caña de azúcar. El nivel de desarrollo y la diversidad en la producción, posicionan a la comunidad en indicadores de SAN y condiciones de vida digna. En la Figura I, se observa el comportamiento del sistema agropecuario, en la zona rural del municipio de San Miguel.

Figura I. Sistema agropecuario, identificado en la zona rural del municipio de San Miguel.

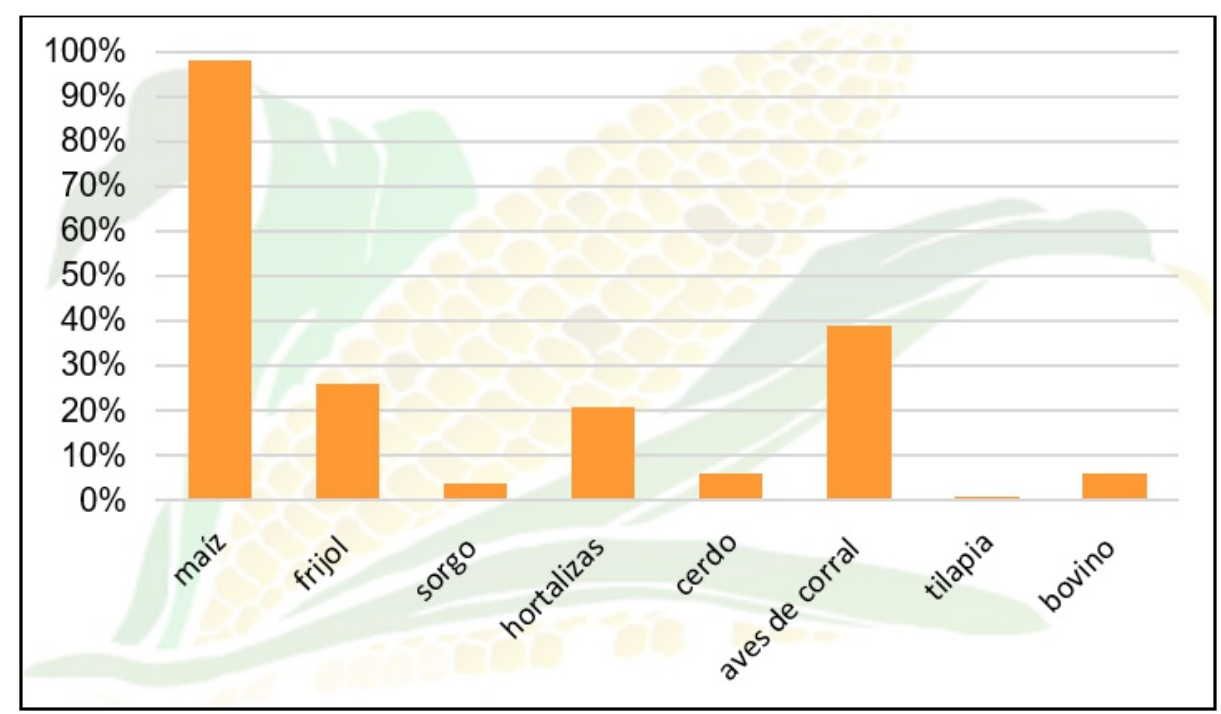

Fuente: Elaboración propia

"El sistema agropecuario en las comunidades rurales del municipio de San Miguel, está dedicado, en su mayor parte, a la siembra de maíz. Los datos recogidos en este estudio indican que en un $98 \%$ de las familias cultivan este grano como medida de seguridad alimentaria. Además, un $39 \%$ posee en sus hogares aves de corral refiriéndose a ello como "gallina, pavo, pato". Los datos indican, también, un porcentaje considerable del $26 \%$ que cultiva frijol, y un $20.8 \%$ que se dedica al cultivo de hortalizas. Los otros rubros son poco explotados, como es el caso del del sorgo, cerdo, tilapia y bovino.

\section{"Composición de la mano de obra familiar}

Las actividades agropecuarias en la zona rural del municipio de San Miguel están ligadas a la época de lluvia. La mayor demanda de fuerza laboral familiar se da a partir del mes de agosto y finaliza con labores de postcosecha en enero y febrero. La Figura 2, recoge datos sobre la cantidad de días que disponen al mes para las labores agropecuarias en cada uno de los rubros identificado en los sistemas agropecuarios en las comunidades. 
Figura 2. Promedio de días invertidos al mes, en mano de obra familiar para iniciativas agropecuarias, en la zona rural del municipio de San Miguel.

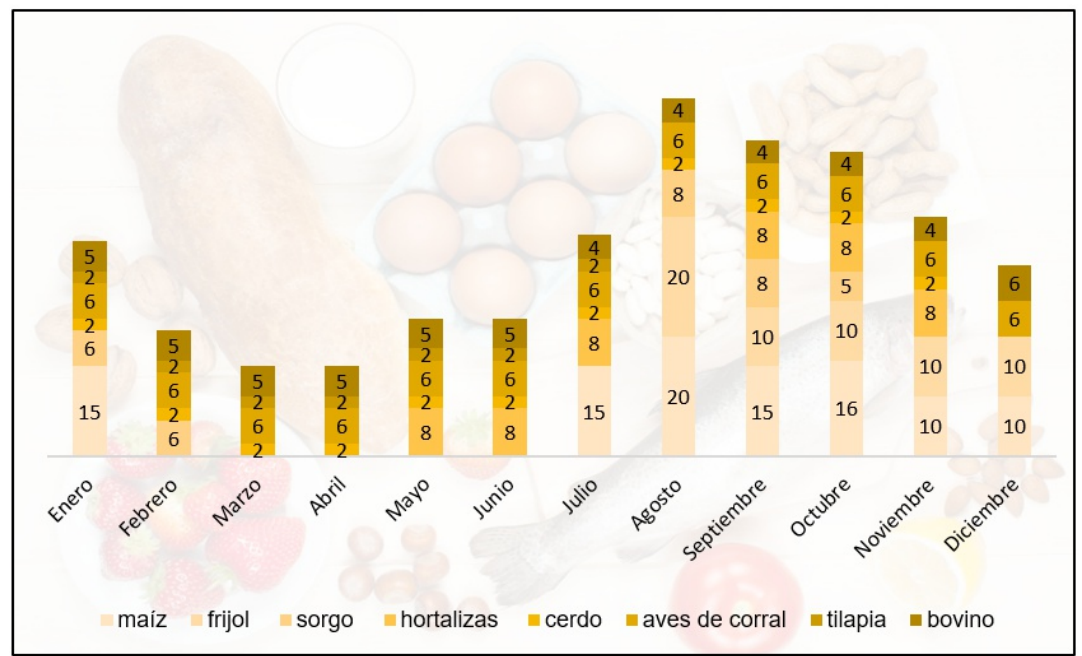

Fuente: Elaboración propia

Al observar los datos en la Figura 2, se puede apreciar que las familias en las comunidades, demandan la mayor cantidad de tiempo en cultivos como el maíz, cuyas actividades de manejo agronómico, inician en la última quincena de julio con la preparación de la tierra y concluyen entre enero y febrero con la cosecha y su posterior almacenamiento o venta de la producción a intermediarios. De igual forma esta época conocida como 'postrera' o 'de verano' para los agricultores, es aprovechado para sembrar los granos básicos para el autoconsumo y venta de excedentes, cuando la cosecha es favorable. Las hortalizas son apenas pequeñas parcelas de chile dulce, tomate, ejote o pipián, cultivadas en época de lluvia debido a que en las comunidades no existen sistemas de riego que permitan actividades agrícolas en época de verano.

De esta forma se puede notar que en las comunidades existe un periodo de tiempo sin mayores ocupaciones agropecuarias. Esto repercute en la dinámica productiva de la comunidad, presentando mayores problemas de alimentación en los meses de marzo a agosto, donde ocurre muchas veces que se dispone de poca disponibilidad de alimentos. En ese sentido las comunidades deben articularse y optar por otras alternativas de medios de vida que les permita mayor disponibilidad y así mejorar sus condiciones de vida.

\section{» Asistencia a la producción agropecuaria}

Por asistencia a la producción agropecuaria se entienden los esfuerzos y estrategias externas para que los pequeños productores dedicados a la agricultura familiar, puedan mejorar sus 
iniciativas productivas agropecuarias a través de incentivos, que pueden ser: insumos agropecuarios, asistencia técnica $\circ$ en dinero en diversos rubros, y con ello tener un adecuado nivel de SAN.

En ese sentido se construyó un Índice de Asistencia a la Producción -en adelante IAP-, definido a partir de tres ítems del cuestionario relacionados con la asistencia a la producción: ¿Qué tipo de ayuda recibes en tus iniciativas productivas?, ¿Qué instituciones te han apoyado? y ¿Cuáles son los rubros en los que te han apoyado? A partir de ello, se crearon tres categorías: IAP-Bajo, IAP-Medio y IAP-Alto, considerando los puntajes obtenidos en cada ítem.

Respecto a las asignaciones de los puntajes, se definió un 1.0 para IAP-Bajo, un 2.0 para IAPMedio y 2.75 IAP-Alto. Para tener un IAP-Alto en las comunidades es necesario obtener diferentes apoyos en las actividades agropecuarias, así como que diversas instituciones orientadas a varios rubros agropecuarios apoyen los procesos de producción, con la finalidad de garantizar el acceso a diversos alimentos. En ese sentido se determinó en porcentajes las categorías de IAP. En la Figura 3, se presenta de forma general y en la Figura 4, aparece por comunidad

Figura 3. Porcentaje de Índice de Asistencia a la Producción

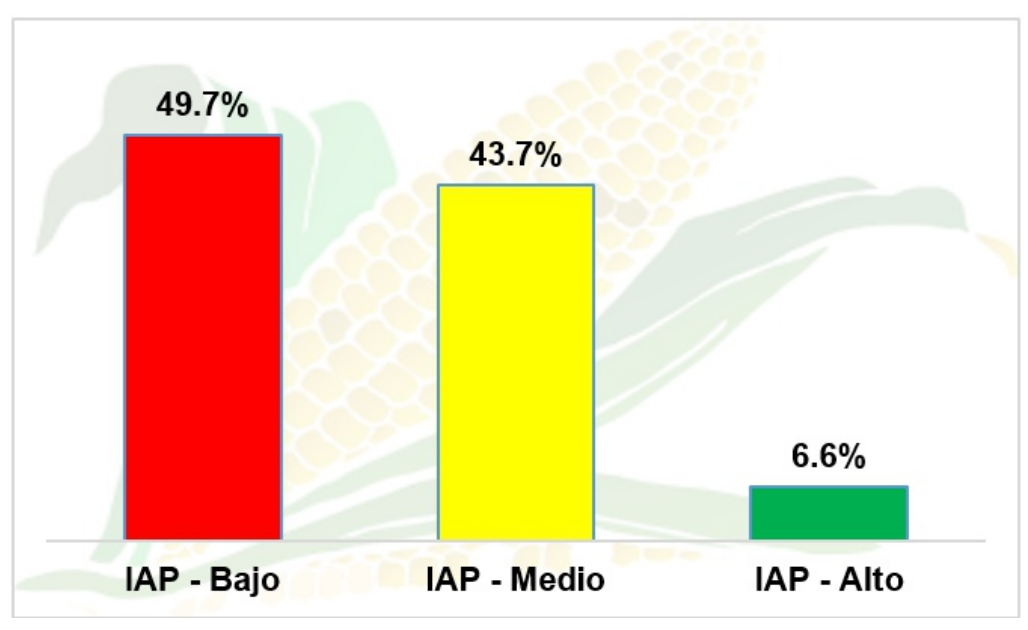

Fuente: Elaboración propia

De los resultados encontrados salen evidencia un IAP - Bajo en un $49.7 \%$, seguido con IAPMedio del $43.7 \%$ y un IAP - Alto de $6.6 \%$. 
Figura 4. Porcentaje de Índice de Asistencia a la Producción en cuatro comunidades del municipio de San Miguel.

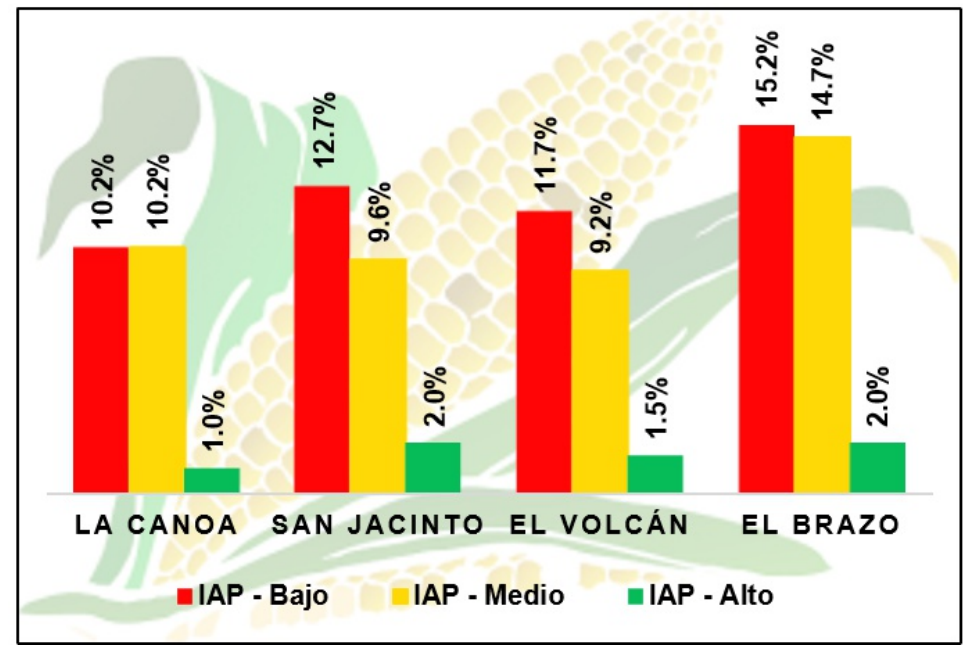

Fuente: Elaboración propia.

Al analizar el IAP por comunidad, resulta que los datos son similares a los observados en la Figura 3, identificando en las comunidades Índices de Asistencia a la Producción bajo y medio en mayores porcentajes, mientras que se observa un IAP-Alto entre $1.0 \%$ y $2.0 \%$ en las cuatro comunidades.

\section{" Índice de Iniciativas Agropecuarias Familiar}

La agricultura familiar está caracterizada por la producción agropecuaria en la familia, que permite la disponibilidad de alimentos en el hogar. Asimismo, para indagar el avance de las comunidades en prácticas de agricultura familiar, se determinó el Índice Iniciativas Agropecuaria Familiar en adelante IIAF, en tres categorías: IIAF-Bajo, IIAF-Medio e IIAF-Alto.

Los resultados son definidos en porcentajes por cada categoría, con la finalidad de evidenciar las actividades agropecuarias en las comunidades que participaron en el estudio. La Figura 5 nos muestra este Índice.

Figura 5. Porcentaje de Índice en Iniciativas Agropecuaria Familiar

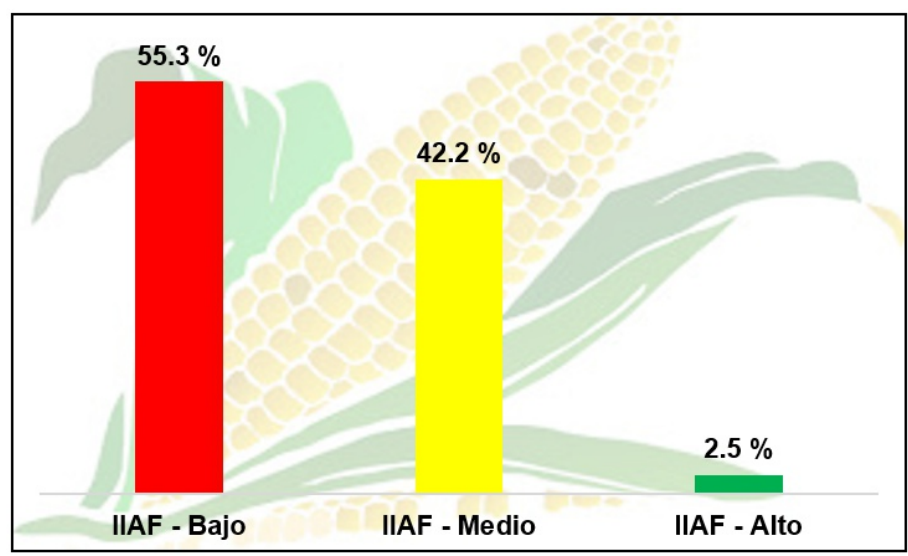

Fuente: Elaboración propia 
Se puede observar que un $55.3 \%$, de las familias tiene un IIAF-Bajo, un $42.2 \%$ tiene un IIAFMedio, y un $2.5 \%$ un IIAF-Alto. Estos datos evidencian un bajo número de iniciativas productivas en las comunidades objeto de estudio, lo que implica dificultades para la disponibilidad de alimentos. En ese sentido es necesario promover un modelo de agricultura familiar que priorice la diversificación de iniciativas agropecuarias.

La productividad agropecuaria define en las comunidades el desarrollo y a su vez contribuye en forma directa a la mejora de la alimentación para las familias. En ese sentido es importante diversificar las iniciativas en las familias, determinar el potencial de trabajo y las capacidades instaladas en las comunidades y aprovechar condiciones para emprender un modelo agropecuario sustentable dinamizador de la economía rural, además de cimentar las bases para lograr el objetivo 2, de los ODS, hambre cero.

\section{»Niveles de SAN en las comunidades}

A partir de la Escala Latinoamericana y Caribeña de Seguridad Alimentaria (ELCSA), los hogares estudiados fueron clasificados en hogares seguros, es decir con un nivel de Seguridad Alimentaria adecuado; hogares con una Inseguridad Alimentaria Leve, hogares con una Inseguridad Alimentaria Moderada, y hogares con una Inseguridad Alimentaria Severa.

Los rangos que aparecen en la clasificación de la Inseguridad Alimentaria, son determinados por el número de respuestas afirmativas en el cuestionario del ELCSA. En la Figura 6, se observan los niveles de Seguridad Alimentaria en hogares solo con adultos.

Figura 6. Niveles de Seguridad Alimentaria en cuatro comunidades del municipio de San Miguel, en hogares solo adultos.

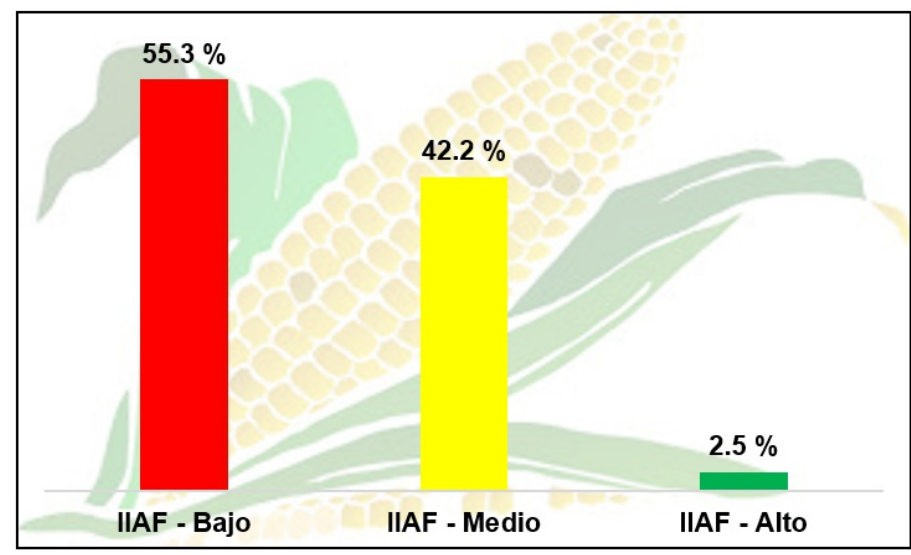

Fuente: Elaboración propia

Según datos mostrado por la ELCSA, existe un $8.7 \%$ de familias en condiciones adecuadas de Seguridad Alimentaria, un 34.6\% hogares con Inseguridad Alimentaria Leve, un $22.8 \%$ con Inseguridad Alimentaria Moderada y un $33.9 \%$ en condiciones de Inseguridad Alimentaria Severo. 
Considerando la importancia que tienen las Iniciativas Productivas Familiares Agropecuarias para la Seguridad Alimentaria en las comunidades, se plantearon las siguientes hipótesis:

\section{Hipótesis I.}

Hi: Los Niveles de Seguridad Alimentaria mejoran a partir de Prácticas de Agricultura Familiar en hogares de cuatro comunidades: La Canoa, San Jacinto, El Volcán y El Brazo.

Para conocer si existe relación entre los niveles de Seguridad Alimentaria y el Índice Iniciativas Agropecuaria Familiar (IIAF) se aplicó la prueba de Chi-cuadrado.

Tabla 2. Prueba de Chi-cuadrado, correlación de IIAF y niveles de Seguridad Alimentaria para hogares con integrantes de solo adultos.

\begin{tabular}{lccc}
\hline Detalles & Valor & gl & $\begin{array}{c}\text { Significación asintótica } \\
\text { (bilateral) }\end{array}$ \\
\hline Chi-cuadrado de Pearson & 8.085 & 6 & 0.232 \\
Razón de verosimilitud & 8.980 & 6 & 0.175 \\
Asociación lineal por lineal & 0.012 & 1 & 0.913 \\
N de casos válidos & 70 & & \\
\hline
\end{tabular}

Fuente: Elaboración propia

El resultado indica un P-valor $>0.05 / 0.232$ indicando que no existe relación entre las Iniciativas Agropecuaria Familiar y los Niveles de Seguridad Alimentaria en los hogares con adultos. Aceptando la hipótesis nula (Ho). En la Figura 7, aparecen los niveles de Seguridad Alimentaria para los hogares que tienen miembros menores de 18 años.

Figura 7. Niveles de Seguridad Alimentaria en cuatro comunidades del municipio de San Miguel, en hogares con menores de edad.

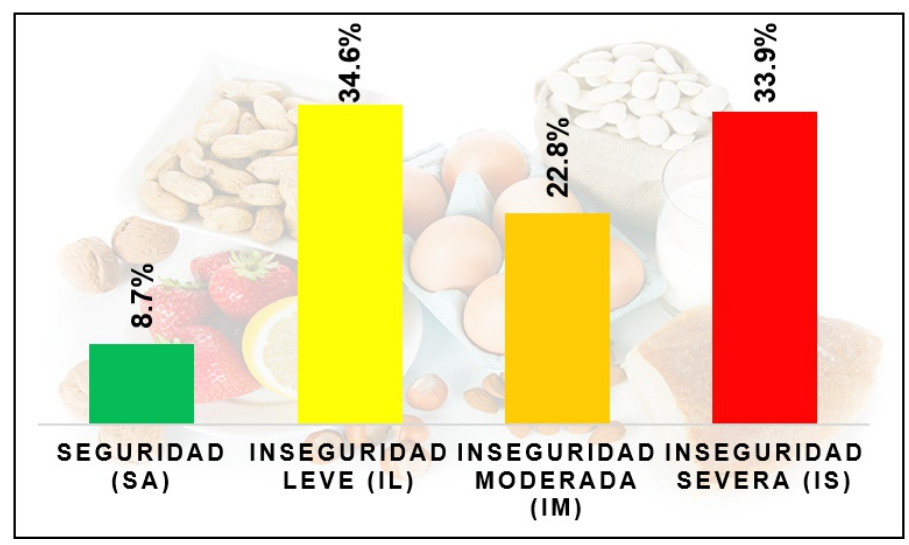

Fuente: Elaboración propia

Este caso se observa un $8.6 \%$ de Seguridad Alimentaria, un $31.4 \%$ de Inseguridad Alimentaria Leve, un $32.9 \%$ Inseguridad Alimentaria Moderada y un $27.1 \%$ de Inseguridad 
Severa. De igual manera, se correlacionó la variable IIAF con los niveles de Seguridad Alimentaria.

Tabla 3. Prueba de Chi-cuadrado, correlación de IIAF y niveles de SAN para hogares con miembros menores de edad.

\begin{tabular}{cccc}
\hline Detalles & Valor & gl & $\begin{array}{c}\text { Significación } \\
\text { asintótica } \\
\text { (bilateral) }\end{array}$ \\
\hline Chi-cuadrado de Pearson & 2.457 & 6 & 0.873 \\
Razón de verosimilitud & 3.040 & 6 & 0.804 \\
Asociación lineal por lineal & 0.175 & $\mathrm{I}$ & 0.675 \\
N de casos válidos & 127 & & \\
\hline
\end{tabular}

Fuente: Elaboración propia

Los niveles de Seguridad Alimentaria no han mejorado a partir de las Prácticas o Iniciativas Agropecuarias Familiar en las comunidades objeto de estudio.

En la siguiente figura se muestra el comportamiento de las respuestas afirmativas en las comunidades para la Escala Latinoamericana y Caribeña de Seguridad Alimentaria (ELCSA).

Figura 8. Porcentaje de respuestas afirmativas de la ELCSA, en cuatro comunidades del municipio de San Miguel.

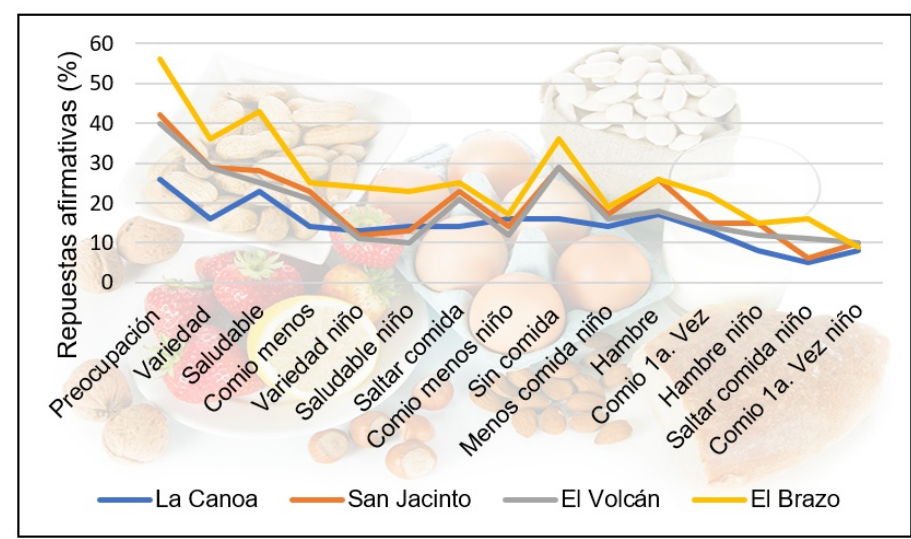

Fuente: Elaboración propia

Es evidente que en todas las comunidades existe similar comportamiento de los datos, en cuanto a preocupación por la falta de alimentos en el hogar, poca variedad de éstos y una alimentación menos balanceada y nutricional. De igual manera, existe un bajo porcentaje que está en condiciones severas de Inseguridad Alimentaria, en donde hay menores que sufren 
hambre.

\section{"Condiciones de vida en las comunidades}

Los rangos definidos de acuerdo a las ponderaciones son: de 0.0 a I.0 ICV-Malo, de 1.10 a 2.30 ICV-Regular, de 2.3I a 2.90 ICV-Buena. Se consultó a las familias de cómo perciben sus propias condiciones en su hogar, en cuanto alimentación, salud, educación, economía y vivienda. Los datos son presentados en la Figura 9.

Figura 9. Porcentaje de percepción de Índice en Condiciones de Vida en cuatro comunidades del municipio de San Miguel.

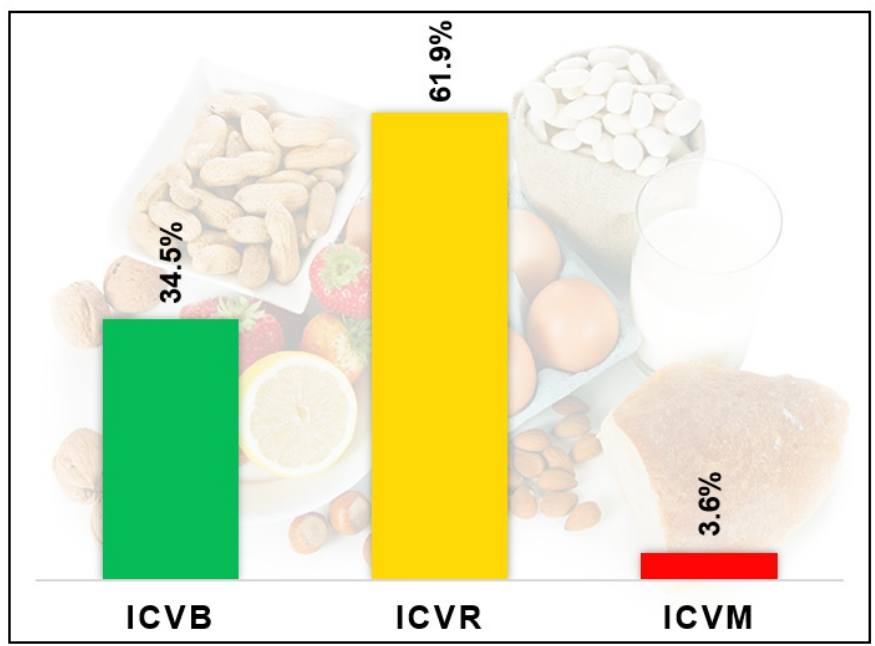

Fuente: Elaboración propia

Con un $61.9 \%$, las familias manifiestan que tienen unas Condiciones de Vida Regular asegurando, que existen dificultades en su economía, salud, vivienda, escolaridad y el acceso a una alimentación adecuada; mientras que un $34.5 \%$ opinaron que se encuentra en Buenas Condiciones de Vida, por último, solo un $3.6 \%$ respondieron que se encuentran en Condiciones de Vida Mala.

Además, el estudio planteó una segunda hipótesis de investigación, con el objetivo de identificar si existe relación de Condiciones de Vida y las Prácticas de Agricultura Familia en las comunidades. En ese sentido se consideró correlacionar el IIAF y el ICV para comprar la Hipótesis 2.

\section{Hipótesis 2.}

Hi: Las iniciativas de agricultura familiar $y$ otros factores externos determinan las condiciones de vida en hogares de cuatro comunidades: La Canoa, San Jacinto, El Volcán y El 
Brazo.

Tabla 4. Prueba de Chi-cuadrado, correlación de IIAF e ICV en cuatro comunidades del municipio de San Miguel.

\begin{tabular}{lccc}
\hline \multicolumn{1}{c}{ Detalles } & Valor & gl & $\begin{array}{c}\text { Significación asintótica } \\
\text { (bilateral) }\end{array}$ \\
\hline Chi-cuadrado de Pearson & .998 & 4 & 0.910 \\
Razón de verosimilitud & 1.185 & 4 & 0.881 \\
Asociación lineal por lineal & 0.606 & $\mathrm{I}$ & 0.436 \\
N de casos válidos & 197 & & \\
\hline
\end{tabular}

Fuente: Elaboración propia

El resultado indica un P-valor $>$ 0.05/0.910 mostrando que no existe relación entre las iniciativas agropecuarias familiar y la percepción de las familias a las condiciones de vida, aceptando la hipótesis nula (Ho). Partiendo de un nivel bajo de IIAF, éste no está contribuyendo a mejorar las condiciones de vida de las familias en las comunidades, los hogares no tienen acceso a una alimentación adecuada ni a condiciones dignas de vivienda, salud, educación, y se percibe, en general, una economía que no permite cubrir las necesidades elementales en el hogar.

\section{Discusión}

A nivel latinoamericano existen y persisten experiencias exitosas en comunidades que practican agricultura familiar, que fomentan mejores condiciones de vida, y que aspiran a mejorar la Seguridad Alimentaria y Nutricional. En ellas, se percibe disponibilidad, acceso, consumo y estabilidad de los alimentos, sentando las bases para el desarrollo comunitario, garantizando y fomentando el derecho a la alimentación adecuada como primer elemento del desarrollo humano.

Bajo este contexto, el estudio tuvo como base la agricultura familiar, el estado de Seguridad Alimentaria y Nutricional y las condiciones de vida en las comunidades La Canoa, San Jacinto, El Volcán y El Brazo, todas pertenecientes al municipio y departamento de San Miguel. Se conocieron las dificultades que predominan en la zona rural en la búsqueda de un desarrollo social pleno, con indicadores de calidad que permitan a las familias a través de sus propios esfuerzos satisfacer sus necesidades básicas.

Suministrados los resultados obtenidos a través de técnicas cuantitativas, se recogieron datos concretos para dar soporte empírico a la pregunta de investigación: ¿De qué manera las 
prácticas de agricultura familiar mejoran la Seguridad Alimentaria y las condiciones de vida en hogares de las comunidades La Canoa, San Jacinto, El Volcán y El Brazo del municipio de San Miguel? Para discutir sobre este enunciado, se introduce un factor elemental: la Asistencia a la Producción, mediante las iniciativas productivas como un componente determinante para mejorar significativamente la producción agropecuaria familiar en las comunidades.

Bajo la anterior lógica, se indagó cuál era el Índice de Asistencia a la Producción en las comunidades. Los resultados evidenciaron un IAP de $49.7 \%$ bajo, $43.7 \%$ medio, y apenas un $6.6 \%$ alto. Es decir, el apoyo principal está basado en un pequeño paquete agrícola, consistiendo por lo general en semillas y fertilizantes químicos. Cuando en realidad la Asistencia a la Producción debe generar las bases para iniciar esfuerzos territoriales para establecer un modelo agropecuario sustentable en todos sus niveles óptimos: tiempo, productividad y rentabilidad.

Por una parte, es importante considerar el planteamiento de Rodríguez Espinosa, y otros, (20I4), "La identificación colectiva de necesidades y la toma conjunta de decisiones en la formulación de proyectos para el desarrollo agropecuario es un proceso que requiere la identificación de metodologías participativas para fomentar el compromiso activo y reflexivo de los productores". En esta dinámica las instituciones deben tomar un rol de mediador de procesos y construcción de esfuerzos con total participación comunitaria, facilitando Asistencia a la Producción Agropecuaria en una dinámica de compromiso social de transformación, acompañadas de políticas públicas que prioricen las condiciones dignas de las familias.

Ahora bien, las prácticas o iniciativas agropecuarias en las comunidades rurales, en su mayoría definen la capacidad para el acceso los alimentos y a otros recursos vitales para el bienestar familiar. En ese sentido, los resultado identificaron que un $55.3 \%$, de las familias tienen un IIAF bajo, mientras que $42.2 \%$ tienen un IIAF medio, y $2.5 \%$ un IIAF alto, y dentro de la lógica razonable, si se tiene una débil asistencia a la producción agropecuaria, no se puede tener diversificación en Iniciativas Agropecuarias Familiar.

La Organización de Las Naciones Unidad para la Agricultura y la Alimentación. FAO (2015), plantea "El nivel y la combinación de insumos y el tipo de tecnologías y sistemas de gestión que se utilicen influyen en gran medida en el nivel de productividad y en el impacto de la producción en los recursos naturales y el medio ambiente. Lograr la "combinación adecuada", que refleje el valor de los recursos naturales y el costo real de los mismos, es esencial para la sostenibilidad". En consecuencia, las prácticas de agricultura familiar en las comunidades rurales del municipio de San Miguel, están subordinadas al cultivo del maíz, 
convirtiéndose este tipo de producción como el único medio de subsistencia en las familias, un modelo productivo agotado, debido a múltiples factores endógenos que menoscaban cada día más las oportunidades de las zonas rurales para emprender iniciativas agropecuarias sustentables.

Existe una complejidad en el tejido social comunitario rural, para articular esfuerzos que implique la promoción de un modelo agropecuario generador de calidad de vida. En ese sentido un aumento de la producción de alimentos es condición necesaria, pero no suficiente, para aumentar la Seguridad Alimentaria. También deben fortalecerse los derechos sobre la tenencia de la tierra Esto resulta ser uno de los elementos más determinantes que limitan un proceso de gestión de un modelo agropecuario que establezca las bases necesarias en el desarrollo comunitario, porque las familias no son propietarias de sus parceles de tierra, principal recurso para la producción (Organización de las Naciones Unidas para la Agricultura y Alimentación. FAO, 2000).

De acuerdo con los resultados de la ELCSA, las comunidades La Canoa, San Jacinto, El Volcán y El Brazo, presentan serios problemas en Seguridad Alimentaria. Los datos señalan de forma generalizada un $\mathbf{8 . 7 \%}$ de familias en condiciones adecuadas de Seguridad Alimentaria, un 34.6 $\%$ hogares con Inseguridad Alimentaria Leve, un 22.8\% con Inseguridad Alimentaria Moderada y un $33.9 \%$ en condiciones de Inseguridad Alimentaria Severo.

La FAO (2007), retomando la Declaración Universal de Derechos Humanos de 1948 proclamó que "Toda persona tiene derecho a un nivel de vida adecuado que le asegure, así como a su familia, la salud y el bienestar, y en especial la alimentación" (FAO, 2007). Es cuestionable que la mayoría de las familias en las comunidades rurales no cuenten con una alimentación adecuada. El concepto de Seguridad Alimentaria y Nutricional parte de un acumulado de condiciones necesarias para que todos los seres humanos, de forma igualitaria $y$ sin ningún tipo de discriminación, existan, desenvuelvan sus capacidades y participen plena y dignamente de la vida en sociedad.

Bajo la anterior perspectiva, surgen ideas claras de cómo potencializar la agricultura familiar y esbozar una estrategia para mejorar la Seguridad Alimentaria y Nutricional en las comunidades. Al respecto Gordillo de Anda (2004), argumenta "Fortalecer el papel de los agricultores familiares está asociado a mejorar su competitividad en los mercados de tierras, productos, trabajo y financiamiento, para lo cual deben disponer de la información necesaria para insertarse en condiciones ventajosas en esos mercados". Por cuanto los retos en el sector rural salvadoreño son muchos, empezando por trabajar los enfoques comunitarios productivos agropecuarios, así como los medios de vida que faciliten trasformaciones 
profundas del sistema mezquino de modelos expropiadores de recursos y de desigualdades sociales que ahogan cada vez más el sector rural.

Al preguntarles a las familias cómo consideran las condiciones de vida en sus hogares, partiendo del acceso a los alimentos adecuados, a la salud, a la educación, a la vivienda y de la economía familiar, un $61.9 \%$ expresó que sus condiciones de vida eran regulares. En el léxico rural, la palabra regular se entiendo como aquellas condiciones que no cumplen con los requerimientos mínimos para llevar una vida plena. En ese sentido, el Centro Latinoamericano para el Desarrollo Social Rimisp (20 I I), señala "La pobreza y la desigualdad son dos problemáticas complejas y diferentes, pero íntimamente relacionadas entre sí. La desigualdad se ve reflejada en diferentes fenómenos políticos, económicos y culturales como la distribución del consumo, la propiedad de la tierra, el acceso a la salud y educación, y la representatividad política".

\section{Conclusión}

A manera de conclusión, se puede afirmar que los niveles de Inseguridad Alimentaria en las comunidades son preocupantes, se tiene $8.6 \%$ con Seguridad Alimentaria; un $31.4 \%$ en Inseguridad Alimentaria Leve, un 32.9.\% con Inseguridad Alimentaria Moderada, y un 27.1\% con Inseguridad Alimentaria Severa en hogares con menores de edad. Estos datos evidencian que las comunidades requieren de estrategias integrales para mejorar los niveles antes descritos. En este contexto, los anteriores datos nos llevan a pensar que aquellas comunidades a las cuales la Alcaldía Municipal de San Miguel y el Ministerio de Agricultura y Ganadería llegan con los paquetes agrícolas, los niveles de Seguridad Alimentaria serán mejores. Para tener parámetros comparativos, se tomó como muestra dos comunidades: La Canoa y El Brazo, en donde la Alcaldía tiene programas como servicios de maquinaria, asesoría agropecuaria e incentivos como fertilizantes y semillas desde hace cuatro años; y las comunidades de San Jacinto y El Volcán, que recién inician con el programa de la alcaldía de San Miguel. Sin embargo, en cuanto a los niveles de SAN no existen diferencias significativas.

En cuanto a las condiciones de vida percibida por jefes o jefas de hogar a partir de las actividades agropecuarias, las familias consideran su alimentación, vivienda, salud y economía familiar entre buena y regular, y en muy bajos porcentajes como mala. En este caso se puede decir con un enfoque cultural, que las familias en las zonas rurales perciben sus condiciones de vida como buena o regular a partir de la condición de tener, al menos, acceso a una alimentación, aunque no sea adecuada, y tener vivienda, aunque no cumpla con condiciones mínimas para generar un ambiente seguro, de contar con una salud que les permita hacer 
sus actividades de rutina, un nivel de escolaridad que les permita leer y escribir y una economía que les permita cubrir algunas necesidades básicas en el hogar.

En cuanto a las interpretaciones de las familias, en la percepción de la agricultura familiar como una herramienta para la mejora de las condiciones de vida en la comunidad, se pueden argumentar diferentes criterios, $y$ es evidente que las familias tienen especial atención a incentivos agropecuarios en las comunidades. Sin embargo, estos incentivos asumen otros roles e incluyen temas de intereses político-partidarios y conflictos de interés. En ese sentido, el avance en el mejoramiento de las condiciones de vida no se percibe de forma tangible Las necesidades y la preocupación por la disponibilidad y el acceso a los alimentos es un denominador común en todos los hogares de las comunidades.

No menos importante es el hecho que las familias de la zona rural en las comunidades asumen sus condiciones de pobreza y desigualdad social de forma pasiva, con resignación de llamarse pobres por vivir en una zona rural. En esa complicidad de múltiples factores, las familias no comprenden la necesidad de gestionar junto a las instituciones con presencia en los territorio, las estrategias de desarrollo comunitario basadas en un proceso participativo y reflexivo, que organice una dinámica social comprometida con un modelo agropecuario moderno, sustentable y amigable con los recursos naturales.

Existen estudios sobre desarrollo comunitario que tienden a centrar el concepto de desarrollo comunitario de forma parcial bajo el significado de "aumento del nivel de vida". Sin embargo, es necesario un análisis más profundo que no tenga en cuenta sólo los planes comunitarios que se ofrecen a la ciudadanía, sino también el proceso participativo que se genera a la hora de formularlos. Entonces, hablar de perspectivas de desarrollo comunitario implica una mirada amplia desde la racionalidad del término desarrollo, y una profunda voluntad política estatal por introducir el tema de la calidad de vida en la población rural como una herramienta de desarrollo.

Es importante señalar que en las comunidades existe un potencial, un capital social que tipifican a las comunidades con la disponibilidad de apoyar propuestas gubernamentales. En ese sentido, no es menospreciable el trabajo de la municipalidad en el territorio y de otras instituciones de gobierno por intentar apoyar iniciativas agropecuarias que dinamicen los territorios en la zona rural para lograr su desarrollo. Sin embargo, los resultados de este trabajo demuestran una meta no alcanzada. Entonces para mejorar el ambiente agropecuario, se requieren otras estrategias como protección del agro a través de leyes que beneficien a productores familiares, seguros y subsidios agropecuarios, y un control del estado para 
incentivar la producción agropecuaria.

\section{Bibliografía}

Centro Latinoamericano para el Desarrollo Social (Rimisp). 20I I. Pobreza y Desigualdad Informe Latinoamericano. Santiago de Chile: s.n., 20II. 978-956-9153-0I-3.

Esquinas, José. 2017. Especular con los alimentos es un crimen, pero es completamente legal. [entrev.] Aurora Moreno Alcojor. Córdoba: Blog. El carro de combate, periodismo independiente "Investigamos lo que consumes", 15 de marzo de 2017.

Gordillo de Anda, Gustavo. 2004. Seguridad Alimentaria y Agricultura Familiar. [En línea] agosto de 2004. [Citado el: 20 de febrero de 2019.] https://repositorio.cepal.org/bitstream/handle//I362/10965/08307/084_es.pdf? sequence.

Hernández Sampieri, Roberto, Fernández Collado, Carlos y Pilar Baptista, Lucio. 2016. Metodología de la Investigación. Sexta. México: Mc Graw Hill, 2016.

Organización de las Naciones Unidas para la Alimentación y la Agricultura. FAO; Fondo Internacional de Desarrollo Agrícola. FIDA y Programa Mundial de Alimentos. PMA. 2018. El estado de la Inseguridad Alimentaria en el mundo. Roma, Italia: s.n., 2015. 978-92-5-308785-3.

Organización de Las Naciones Unidad para la Agricultura y la Alimentación. FAO. 20I5. Construyendo una visión común para la agricultura y alimentación sostenibles. [En línea] 20I5. [Citado el: 28 de febrero de 2019.] http:// www.fao.org/3/a-i3940s.pdf. 978-92-5-30847I-5.

Organización de las Naciones Unidas para la Agricultura y Alimentación. FAO. 2000. El estado mundial de la agricultura y la alimentación. [En línea] 2000. [Citado el: 20 de febrero de 2019.] http://www.fao.org/3/x4400s/ x4400s.pdf. 92-5-304400-4.

Organización de las Naciones Unidas para la Agricultura y Alimentación. FAO. 2007. El Derecho Humano a la Alimentación. [En línea] 2007. [Citado el: 20 de febrero de 2019.] http://www.fao.org/3/a-a I60 Is.pdf.

Organización de las Naciones Unidas para la Alimentación y la Agricultura. FAO. 20I4. Agricultores Familiares, Alimentar al mundo, cuidar al planeta. [En línea] abril de 20l4. [Citado el: 8 de marzo de 2019.] http:// www.fao.org/assets/infographics/FAO-Infographic-IYFFI4-es.pdf.

Organización de las Naciones Unidas. ONU. 2017. Informe de los Objetivos de Desarrollo Sostenible 2017. Nueva York: s.n., 2017. 978-92-I-361717-5.

Padua, Jorge, y otros. 1979. Técnicas de investigación aplicadas a las ciencias sociales. Tercera. 1996. México : Fondo de Cultura Económica (FCE), 1979. págs. 40-45. ISBN 968-16-0288-9.

Rodríguez Espinosa, Holmes y Ramírez Gómez, Carlos Julián. 2014. Abordaje metodológico para formulación participativa de planes de asistencia técnica agropecuaria con enfoque territorial. [En línea] 20I4. [Citado el: 21 de febrero de 2019.] https://revistas.unal.edu.co/index.php/acta_agronomica/article/viewFile/45I62/5I488. 0I202812 .

Sierra Bravo, Restituto. 200I. Técnicas de Investigación Social. Decimocuarta. Madrid: Paraninfo, 200 I. 